
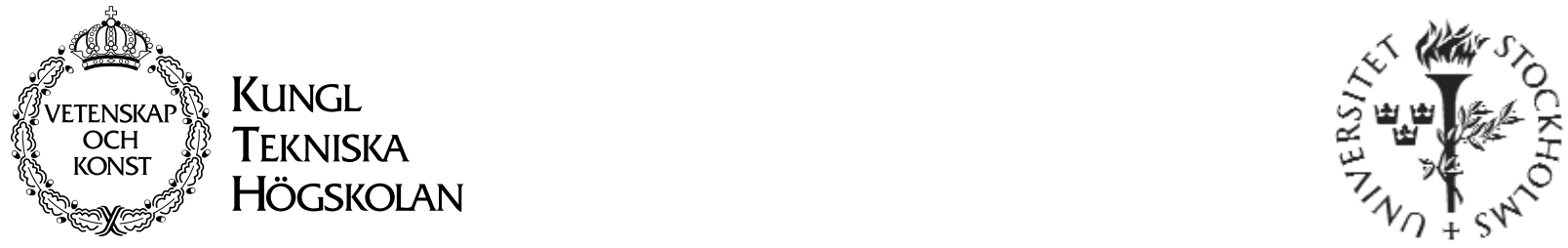

CID-121 • ISSN 1403-0721 • Department of Numerical Analysis and Computer Science $\bullet$ KTH

\title{
The ISO Approach to the Development of Ergonomics Standards for Accessibility
}

Jan Gulliksen, Susan Harker and John Steger. 


\section{Författare: Jan Gulliksen, Susan Harker and John Steger}

The ISO Approach to the Development of Ergonomics Standards for Accessibility.

Report number: CID-121

ISSN number: ISSN 1403-0721 (print) 1403-073X (Web/PDF)

Publication date: 2001

E-mail of author: jan.gulliksen@hci.uu.se

URL of author: http://cid.nada.kth.se

Reports can be ordered from:

CID, Centre for User Oriented IT Design

NADA, Deptartment of Numerical Analysis and Computer Science

KTH (Royal Institute of Technology)

SE-100 44 Stockhom, Sweden

Telephone: +46 (0) 87909100

Fax: + 46 (0) 87909099

E-mail: cid@nada.kth.se

URL: http://cid.nada.kth.se 


\title{
CHAPTER NUMBER
}

\section{ISO 16071 Ergonomics of human- system interaction - guidance on software accessibility}

\author{
Jan Gulliksen ${ }^{1,2}$ (Jan.Gulliksen@hci.uu.se), \\ Susan Harker³ (S.D.Harker@lboro.ac.uk), \\ John Steger ${ }^{4}$ (jsteger@us.ibm.com),
}

${ }^{1}$ Department of HCI, Information Technology, Uppsala University, Uppsala, Sweden

${ }^{2}$ Center for user-oriented IT-design (CID), Royal Institute of Technology, Stockholm, Sweden

${ }^{3}$ Loughborough Institute of Technology, United Kingdom

${ }^{4}$ IBM, United States of America

\begin{abstract}
This chapter reports on the work currently performed by the International Organization for Standardization (ISO) on accessibility of human-computer interfaces. ISO Technical Specification 16071 "Ergonomics of human-system interaction - guidance on software accessibility" will be published by the end of 2000.
\end{abstract}

\section{INTRODUCTION}

With the increasing interest in designing usable systems and the increasing the level of accessibility for people with specific potential requirements, the International Organisation for Standardisation has been conducting work resulting in a ISO Technical Specification 16071 Ergonomics of human-system interaction guidance on software accessibility.

Here I would like an introduction that stresses the importance of addressing the growing population of users who need extra support to be able to handle computer support. How big are these groups? I know that we can find information in the Nordic guidelines.

What are the incitements to support this type of work? E.g. referencing ADA (American Disabilities Act)

INTERNATIONAL ORGANISATION FOR STANDARDIZATION (ISO) 
The International Organization for Standardization (ISO) is a worldwide federation of national standards bodies from some 130 countries, one from each country. ISO is a non-governmental organisation established in 1947. The head office is in Geneva. The mission of ISO is to promote the development of standardisation and related activities in the world with a view to facilitating the international exchange of goods and services, and to developing co-operation in the spheres of intellectual, scientific, technological and economic activity. ISO's work results in international agreements, which are published as International Standards.

ISO standards are developed according to the following principles:

- Consensus - The views of all interests are taken into account: manufacturers, vendors and users, consumer groups, testing laboratories, governments, engineering professions and research organisations.

- Industry-wide - Global solutions to satisfy industries and customers worldwide.

- Voluntary - International standardisation is market-driven and therefore based on voluntary involvement of all interests in the marketplace.

The international standard described in this document is provided by ISO TC 159/SC 4/WG 5 "Software ergonomics and human computer dialogues". WG 5 is the group behind the software parts of ISO 9241 "Ergonomic requirements for office work with visual display terminals (VDTs)", that is parts 10 through 17. WG 5 is also currently preparing ISO 14915 "Multimedia User Interfaces".

Guidance on Accessibility of Human Computer Interfaces - ISO 16071 is not an international standard, but a Technical Specification (TS). A TS is a new format for ISO documents presented at first in 1998. It is a normative document representing the technical consensus within an ISO committee. The purpose is that a technical specification within a period of six years should be converted into an international standard or be withdrawn.

\section{THE INTRODUCTION TO ISO TS 16071}

The purpose of ISO Technical Specification 16071 is to provide guidance to developers on designing human-computer interfaces which can be used with as high level of accessibility as possible. Designing human computer interactions to increase accessibility promotes increased effectiveness, efficiency, and satisfaction for people who have a wide variety of capabilities and preferences. Accessibility is therefore strongly related to the concept of usability (ISO 9241 Part 11 - Guidance on usability).

The most important methodological approaches to increase the accessibility of a given human-computer interface are:

- Task-oriented design of user interfaces.

- Customisation

- The use of human centred design principles (ISO 13407 - Human centred design process for interactive systems).

- Individualised user instruction and training.

The focus of ISO TS 16071 is development of human computer interactions to 
systems and products that are intended for use by the widest range of people with special needs. An important part of a human centred design process for accessible systems is to develop human-computer interfaces to meet accessibility goals that can be quantitatively evaluated for a specific user or user category in a specified context of use.

The ISO Technical Specification 16071 is based mainly on the prevalent knowledge of individuals with sensory and/or motor impairments in an office work context. However, accessibility is an attribute that affects a large variety of capabilities and preferences of human beings. These different capabilities may be the result of age, disease, and/or disabilities. Therefore, accessibility addresses a widely defined group of users including:

- $\quad$ people with physical, sensory and cognitive impairments by birth or acquired during life

- elderly people who can benefit from new IT products and services but experience reduced physical, sensory and cognitive abilities

- people with temporary disabilities such as a person with a broken arm or someone who forgot her glasses

- people who are experiencing difficulties in certain situations, such as a person who works in a noisy environment or has both hands occupied by other work.

Having a disability should be regarded as a natural element of the life cycle. Everyone can expect, during some period of life, to be affected by circumstances that make the access to ad use of IT systems products and services difficult.

ISO TS 16071 recognises that some users will always need assistive devices to use a system. Therefore, it includes the capability of a system to connect and interact successfully with assistive technologies in the concept of accessibility.

ISO TS 16071 provide guidance for system design, appearance and behaviour. The guidance will allow software to be used by as broad an audience as possible. In addition, guidance will be provided on designing software to integrate as effectively as possible with common assistive technologies (e.g., speech synthesisers, Braille input and output devices) when they are available. Incorporating accessibility features early in the design process is relatively inexpensive compared to the cost of modifying products to become accessible.

ISO TS 16071 addresses the increasing need to consider the social and legislative demands to ensure accessibility by removing barriers that prevent them from participating in life activities including the use of environment, services, products, and information. Designing software user interfaces for accessibility increases the number of people who can use computer systems by taking into account the varying physical and sensory capabilities of user populations. Designing for accessibility benefits disabled users by making software easier for them to use or making the difference in whether they are able to use the software at all.

Many accessibility features also benefit users who are not handicapped by enhancing usability and providing additional customisation possibilities. They may also help to overcome temporary deficits (e.g., a broken arm or hand). ISO TS 16071 benefits designers and suppliers by expanding the number of potential users (and thus sales for their product) and often by making the product compliant with legal requirements for accessibility. It benefits companies buying software by 
expanding the number of employees who may use the software.

Accessibility may be provided by a combination of both hardware and software. Assistive technologies typically provide specialised input and output capabilities not provided by the system. Software examples include on-screen keyboards that replace physical keyboards, screen magnification software that allows users to view their screen at various levels of magnification, and screen reading software that allows blind users to navigate through applications, determine the state of controls, and read text via text to speech conversion. Hardware examples include head-mounted pointers that replace mice and Braille output devices that replace a video display. There are many other examples not listed here. When users provide add-on assistive software and/or hardware, usability is enhanced to the extent that systems and applications integrate with those technologies. For this reason, operating systems may have to provide "hooks" or other features to allow software to operate effectively with add-on assistive software and hardware as recommended in these guidelines. If systems do not provide support for assistive technologies, the probability increases that users will encounter problems with compatibility, performance, and usability. At the same time, if software applications do not use system provided mechanisms (such as customisation for colour, font, and audio, or system routines for keyboard navigation and text input), then users can find their access blocked.

\section{SCOPE OF THE ISO 16071}

ISO Technical Specification 16071 provides guidance on design of accessible (work, home, education) software. It covers issues associated with designing accessible software for the widest range of visual, hearing, motor and cognitive abilities, including people who are elderly and temporarily disabled. This Technical Specification addresses software considerations for accessibility that complements general usability design covered by ISO 9241 and ISO 13407.

The Technical Specification covers accessibility of computer operating systems and applications. While it does not address all accessibility issues of platforms and application domains such as web pages, multimedia, Personal Digital Assistants (PDA), and kiosks, many of the recommendations are applicable in these areas. It does not apply to software used primarily for entertainment purposes (i.e., games). It does not provide recommendations for the design of hardware.

The Technical Specification is aimed at reducing the need for add-on assistive hardware and software technologies while promoting increased usability of systems in combination with assistive technologies, when they are required. It does not cover the behaviour or requirements for assistive technologies themselves (including assistive software).

\section{DEFINING ACCESSIBILITY IN RELATION TO USABILITY}

Our experience from the development and use of ISO 9241 part 11 is the benefits of having a clear and succinct definition of the concept. ISO 9241:11 defines 
usability as:

The extent to which a product can be used by specified users, to achieve specified goals, with effectiveness, efficiency and satisfaction, in a specified context of use.

The advantage of such a definition is both that it defines usability to be a quantitatively measurable concept. It also emphasises the non-functional demands of usability that is so important for the final interpretation of the concept.

On the other hand we would want to define accessibility in relation to usability as a measurable entity. Therefore ISO TS 16071 define accessibility as:

The usability of a product, service, environment or facility by people with the widest range of capabilities.

This definition implicitly indicates that it is measurable through its relation to usability. However, it is extended to cover more than just products (as in the definition of usability), covering service, environment or facility. This definition can be compared to the less precise definition from HFES 200 Software User Interfaces - Accessibility:

The set of properties that allows a product, service or facility to be used by people with a wide range of capabilities, either directly or in conjunction with assistive technologies. Although "accessibility" typically addresses users who have a disability, the concept is not limited to disability issues.

This definition suggests accessibility as of a binary nature, i.e. either a product is accessible or not. This does not correspond with essence of the specific guidelines in the document, why we suggest sharpening the definition to be quantitatively measurable. As a comparison, the World Wide Web Consortium $\left(\mathrm{W}^{3} \mathrm{C}\right)$ define accessibility in the following way:

Content is accessible when it may be used by someone with a disability.

Measurable accessibility should be measured in terms of users, task, context and products. To be able to compare the accessibility one must only vary one of these terms at a time, e.g.:

Accessibility $_{1}=\mathrm{f}\left(\right.$ user $_{1}$, task $_{1}$, context $_{1}$, product $\left._{1}\right)$

Accessibility $_{2}=\mathrm{f}\left(\right.$ user $_{1}$, task $_{1}$, context $_{1}$, product $\left._{2}\right)$

\section{RATIONALE AND BENEFITS OF ISO 16071}

Accessibility is an important consideration in the design of products, systems, environments and facilities because if effects the usability of people with the widest possible range of capabilities.

Accessibility can be improved by incorporating features and attributes known to benefit the users with specific special requirements. In order to determine the 
Type in Book Title Here

achieved level of accessibility, it is necessary to measure the performance and satisfaction of users working with a product or interacting with an environment. Measurements of accessibility is particularly important in view of the complexity of the interactions with the user, the goals, the task characteristics and the other elements of the context of use. A product, system, environment or facility can have significantly different levels of accessibility when used in different contexts.

Planning for accessibility as an integral part of the design and development process involves the systematic identification of requirements for accessibility including accessibility measurements and verification criteria within the context of use. These provide design targets that can be the basis for verification of the resulting design.

The approach adopted in ISO Technical Specification 16071 has benefits, which include:

- The framework can be used to identify the aspects of accessibility and the components of the context of use to be taken into account when specifying, designing or evaluating the accessibility of a product.

- The performance (effectiveness and efficiency) and satisfaction of the users can be used to measure the extent to which a product, system, environment or facility is accessible in a specific context.

- Measures of the performance and satisfaction of the users can provide a basis for the comparison of the relative accessibility of products with different technical characteristics, which are used in the same context.

- The accessibility planned for a product can be defined, documented and verified (e.g., as part of a quality plan).

\section{TARGET AUDIENCE}

Who will be using such an ISO document? Today, many organisations have user centred design groups within the organisations to take the responsibility for accessibility issues.

ISO Technical Specification 16071 serves the following types of users:

- the user interface designer, who will apply the guidance during the development process.

- the developer, who will apply the guidance during design and implementation of system functionality.

- the buyer, who will reference the Technical Specification during product procurement.

- evaluators, who are responsible for insuring products meet the recommendations of the Technical Specification.

- designers of user interface development tools and style guides to be used by interface designers.

The ultimate beneficiary of this Technical Specification will be the end-user of the software. Although it is unlikely that the end-users will read the Technical Specification, its application by designers, developers, buyers and evaluators should provide user interfaces that are more accessible. The guidelines concern the development of software for user interfaces, however, those involved in designing 
the hardware aspects of user interfaces may also find them useful.

Here is one of the major problems that I think we have observed and that I would want to discuss. How does the reader find the ISO-standard that is applicable to them in a specific situation? One-stop-shopping could be achieved if all the standards within ergonomics could be collected under one ISO-number. Alternatively one could create a technical report with the purpose of explaining the different standards and their interrelationship and possible use.

I could also fancy discussing the use of checklists to communicate knowledge.

The importance of making the presentation of the material attractive - today many people regard the standards as so boring that one could die...

\section{WHAT TYPES OF CAPABILITIES DO WE WANT TO ADDRESS?}

The user characteristics of people with any given disability vary significantly just as any other heterogeneous population. The descriptions contained here provide only an outline of the issues typically encountered by individuals with various disabilities, and do not constitute a comprehensive list. People may concurrently experience more than one of the disabilities outlined below. The needs of people who have such combinations of disabilities are covered in several cases by the overlap across guidelines.

The following capabilities are specifically addressed in this standard

- users who are blind

- users who have low-vision

- users who are deaf

- users who have hearing impairments

- users who have physical impairments

- users who have cognitive impairments

- users who are elderly

- users who have temporary disabilities

- users who have multiple disabilities

- users who have environmental disabilities

\section{Issues commonly encountered by users who are blind}

The primary issue for users who are blind is how to obtain information provided by visual presentation, how to navigate among objects on screens, how to identify those objects, and how to control focus, navigation, and other functions via the keyboard.

Many people who are blind from birth learn Braille, and many who become blind later in life often rely on additional auditory methods to obtain information.

Many users who are blind often have some vision and primarily interact with computers through "screen readers" -- assistive software that can provide spoken or 
Braille information for windows, controls, menus, images, text and other information typically displayed visually on a screen.

Considerations for these users follow from the characteristics of interactions mediated by screen readers. To the extent that interactions depend on understanding a spatial metaphor for navigation or seeing a graphically represented objects, users who are blind are more likely to encounter difficulties, and normally keyboard navigation is an essential function.

In addition, because many users who are blind are reading screens by means of synthesised speech output, they may find it difficult or impossible to attend to auditory outputs that occur while they are reading.

\section{Issues commonly encountered by users who have low-vision}

The issues commonly faced by users who have low-vision include loss of visual acuity, colour perception deficits, impaired contrast sensitivity, and loss of depth perception.

People who have low-vision use varying means of increasing the size, contrast, and overall visibility of visual displays depending upon their visual needs. Common assistive technologies include use of oversized monitors, large fonts, high contrast, and hardware or software magnification to enlarge portions of the display.

When interacting with computers, these users may not detect size coding, have difficulty with font discrimination, and encounter difficulties locating or tracking interface objects such as pointers, cursors, drop targets, hot spots, and direct manipulation handles.

Additionally, both blind and users who have low vision experience difficulties when required to read very small displays, such as those on printers, copiers, ticket machines and ATMs.

\section{Issues commonly encountered by users who are deaf or hard of hearing}

Users who are deaf or hard of hearing who retain some functional hearing include the inability to discriminate frequency changes, decreased frequency range and dropout, difficulties localising sounds, and difficulty picking up sounds against background noise.

Users who are deaf or hard of hearing may or may not use electronic hearing aids, depending on the nature and extent of the hearing impairment. If it is available in the operating system, they may use the "ShowSounds" feature that notifies software to present audio information in visual form.

When interacting with computers, these users may have trouble hearing sounds of certain frequencies, or of low volume. Sound customisation is key to providing them with access.

\section{Issues commonly encountered by users who are deaf}


In addition to a general inability to detect auditory information, the issues commonly faced by users who are deaf may include the inability to produce speech recognisable by voice input systems, and experience with a national language only as a second language (sign language often being the primary language for people who are born deaf or who become deaf at an early age).

If it is available, users who are deaf will typically use the "ShowSounds" feature that notifies software to present audio information in visual form.

When interacting with computers, these users will encounter problems if important information is presented only in audio form. Many of these issues apply to any user in contexts where sound is masked by background noise (e.g., machine shop floor) or where sound is turned off or cannot be used (e.g., a library).

\section{Issues commonly encountered by users who have physical impairments}

The issues commonly faced by users who have physical impairments often follow from physical limitations including poor co-ordination, weakness, difficulty reaching, and inability to move a limb.

Users with physical impairments may or may not use assistive technologies and the variety of hardware and software they employ is too large to describe in detail in this space. A few examples, however, include eye-tracking devices, onscreen keyboards, speech recognition, and alternative pointing devices.

Some users may have difficulty directly manipulating objects, using modifier keys, using pointing devices, and performing actions that require precise movement or timing. Other users may have tremors that cause difficulty in moving to a target. The extreme variation in needs and capabilities among this user population means that customisation of input parameters and timing is extremely important for effective access.

\section{Issues commonly encountered by users who have cognitive impairments}

The issues commonly encountered by users who have cognitive disabilities involve difficulties receiving information, processing it, and communicating what they know. People with these impairments may have trouble learning new things, making generalisations and associations, and expressing them selves through spoken or written language. Attention deficit hyperactivity disorders make it difficult for a person to sit calmly and give full attention to a task.

The issues commonly faced by users who have dyslexia are difficulties reading text that is presented in written form and difficulties in producing written text.

Reading difficulties is best supported by having the text that is highlighted and read out loud or by providing "easy-reading" versions of the texts. Users without reading difficulties also benefit from easy-reading versions of written text.

Providing support of synthetic speech for what is to be written best supports writing difficulties. 


\section{Issues commonly encountered by users who are elderly}

Elderly users are progressively limited in their ability to use and access humancomputer interfaces due to the multiplicative effects of combinations of visual, hearing, cognitive, and motor impairments that to varying degrees might come with increasing age.

Sometimes the awareness of diminishing capabilities is a concern for elderly users. Therefore, built-in accessibility of products contributes to removing the stigma of special aids or modifications. Elderly persons do not want to have their age regarded as a disability.

\section{Issues commonly encountered by users who have temporary disabilities}

Temporary disabilities are often of a physical nature (e.g. broken arm). These users seldom adopt efficient skills in learning to cope with their disability. It is therefore important to make the accessibility features for these disabilities easy to find and learn to master.

Temporary disabilities might also be caused by repetitive strain caused by poor ergonomics_and intensive use_of the computer system. It is then important that this injury can be relieved through support that can be provided to the user via the system.

For example, improving the design of the laptop computer so that it can be opened with one hand also increases the usability for all users.

\section{Issues commonly encountered by users who have multiple disabilities}

There are not just a few categories of disabilities, rather the range of different accessibility needs varies just as the combinations of certain degrees of disabilities are a fact. For example, an individual with a cognitive impairment might also have low vision.

Several of the guidelines for addressing a specific disability might be contradictory. For example, auditory output of written text is not a support for the deaf blind. It is therefore important that the support for these forms of multiple disabilities is individualised for the specific user and task.

\section{Issues commonly encountered by users who have environmental disabilities}

Environmental disabilities occur when specific features of the work environment cause difficulties in perceiving signals from the computer. Such situations include difficulties hearing signals from the computer, working in a noisy environment. These situations must be regarded as disabling the user to fulfil the task with the aid of the computer. Although this may not be a software requirement, the immediate solution should be to improve the environment, and, in situations where this can not be made, such as airports, provide redundant presentations of essential information. 


\section{WAI GUIDELINES}

The $\mathrm{W}^{3} \mathrm{C}$ has provided one of the most commonly used documents on accessibility. The ISO working group had quite a discussion on whether the material could be regarded as stable and how a standard could reference guidelines that merely are provided on-line. The group went through all of the guidelines provided in $\left[\mathrm{W}^{3} \mathrm{C}, 1999\right]$ and grouped them into the following categories;

- guidelines that are already covered by ISO 9241

- guidelines that are not of a general nature

- guidelines that should be included in the document.

The following decisions were made...

\section{SUMMARY AND POSSIBLE FUTURE DEVELOPMENT EFFORTS}

ISO TS 16071 Ergonomics of human-system interaction - guidance on software accessibility will be published by the end of 2000 . The intention of the group is to move the document further and pursue the work to be an international standard.

\section{ACKNOWLEDGEMENTS}

The authors wish to acknowledge the entire subgroup on accessibility from ISO Technical committee 159/Sub committee 4/Working Group 5. The input provided by Human Factors and Ergonomics Society in terms of their outline of the accessibility part of ANSI 200 is a significant contribution to the making of the technical specification.

\section{REFERENCES}

International Organization for Standardization (1998) ISO 9241 Software ergonomics with visual display terminals (VDTs), Part 11 Guidance on Usability. International standard. (Switzerland: International Organization for Standardization)

International Organization for Standardization (1999) ISO 13407 Human centred design process for interactive systems. International standard. (Switzerland: International Organization for Standardization)

International Organization for Standardization (2000) ISO 16071 Ergonomics of human-system interaction - guidance on software accessibility. Technical Specification. (Switzerland: International Organization for Standardization)

\section{Userfit}

Thorén, C. (ed.) (1998). Nordic Guidelines for Computer Accessibility. $2^{\text {nd }}$ ed., (Vällingby: Noordic Cooperation on Disability). 
World Wide Web Consortium $\left(\mathrm{W}^{3} \mathrm{C}\right)$, (1999) WAI - Web content accessibility guidelines 1.0, May 5, 1999. 